\title{
Investigación de la interacción matriz/refuerzo en materiales compuestos AA6061/partículas Ti-Al mediante análisis de imagen
}

\author{
N. MARTÍNEZ, D. BUSQUETS V. AMIGÓ, Ma D. SALVADOR, C. FERRER \\ Universidad Politécnica de Valencia, España. \\ Departamento de Ingeniería Mecánica y de Materiales. Universidad Politécnica de 46022-Valencia, España.
}

\begin{abstract}
La presente comunicación compara la estabilidad que presentan diferentes refuerzos intermetálicos ( $\mathrm{Ti}_{3} \mathrm{Al}$ y TiAl) adicionados a una matriz de aluminio (AA6061), empleándose para ambos refuerzos diferentes contenidos del mismo. El método de fabricación elegido combina una vía pulvimetalúrgica con un proceso secundario de extrusión en caliente. Para estudiar la interacción del refuerzo con la matriz, se llevan a cabo tratamientos térmicos de solubilización en estufas con atmósfera controlada, a una temperatura de $530^{\circ} \mathrm{C}$ y variando el tiempo entre 0 y 24 h. Se utiliza la microscopía electrónica de barrido (MEB) para el estudio microestructural de los compuestos, calculándose el espesor y la velocidad de crecimiento de las capas de reacción mediante técnicas de análisis de imagen. La estequiometría de las capas formadas se determina mediante microanálisis por energías dispersivas de rayos X (EDX).
\end{abstract}

Palabras clave: compuestos, aluminio, partículas intermetálicas, interacción matriz/refuerzo, análisis imagen.

Image análisis study of matrix reinforcement interaction on AA6061 / Ti-Al particle reinforced composites

In this paper is compared the stability of different contents of intermetallic particles $\left(\mathrm{Ti}_{3} \mathrm{Al}\right.$ and $\mathrm{TiAl}$ ) in an aluminium alloy (AA6061). A powder metallurgy route combined with a secondary hot extrusion process, was followed to fabricate the composites. The matrix/ reinforcement interaction was enhanced by solubilization heat treatments at temperatures ranging form 470 to $530^{\circ} \mathrm{C}$ and times from 1 to 24 h. Scanning electron microscopy was employed to study the microstructure of the composites. The reaction layer thickness and its growing speed were determined by image analysis. The stoichiometric composition was obtained by dispersive energy diffraction of $X$ ray.

Keywords: aluminium matrix composites, intermetallic particles, matrix/reinforcement interaction, image analysis.

\section{INTRODUCCIÓN}

El empleo de materiales compuestos ha aumentado en los últimos años debido a la mejora de propiedades que presentan en comparación con los materiales más convencionales. Presentan una mayor resistencia específica y mayor resistencia al desgaste así como una mayor temperatura de servicio.

En la actualidad los materiales compuestos de matriz de aluminio son de especial interés, habiéndose estudiado en profundidad los sistemas que incorporan partículas de carburo de silicio y alúmina (1-5). La incorporación de nuevos tipos de partículas de refuerzo es de especial interés a fin de mejorar las propiedades finales del compuesto así como evitar algunos de los inconvenientes de la utilización de partículas cerámicas. El empleo de partículas intermetálicas (aluminiuros de titanio o niquel) como refuerzos adecuados para el aluminio, fue propuesta en 1983 por Yamada y Unakoshi (6).

En el presente, los principales obstáculos para la aplicación de los materiales compuestos son su alto precio así como la posible reacción química en las interfaces matriz/refuerzo derivadas del procesado a altas temperaturas $(7,8)$. Esta reacción química conlleva la formación de capas de reacción en las interfaces matriz/refuerzo las cuales modifican las características macroscópicas, mecánicas y térmicas del compuesto (9). El conocimiento de estos mecanismos de reacción es de vital importancia para controlar los procesos de fabricación de los compuestos así como para conocer el efecto que tienen los tratamientos térmicos realizados a temperaturas superiores a $300^{\circ} \mathrm{C}$ en estos compuestos (10).

La adquisición digital de imágenes y su análisis son dos herramientas fundamentales en muchos campos. La investigación relacio- nada con los materiales compuestos requiere la utilización efectiva de los métodos de análisis de imagen para la obtención de determinados valores numéricos y descripción de algunos aspectos de los mismos (11). Así recientemente se está haciendo uso de estas técnicas para caracterizar las interfaces matriz/refuerzo de los materiales compuestos.

\section{PARTE EXPERIMENTAL}

Para la fabricación de los compuestos se ha empleado una matriz de aluminio, la aleación AA6061 y fracciones en volumen del 5, 10 y $15 \%$ de partículas intermetálicas de $\mathrm{Ti}_{3} \mathrm{Al}$ y TiAl obtenidas por atomización y solidificación rápida mediante argón. Aunque los detalles de los polvos de partida se recogen con más detalle en trabajos anteriores presentados por el grupo (12), cabe destacar que estas partículas tienen morfología esférica, un tamaño de partícula inferior a $50 \mu \mathrm{m}$ y no presentan ningún tipo de oxidación superficial.

El proceso de obtención de los compuestos ha combinado una vía pulvimetalúrgica con un proceso de extrusión en caliente. Los detalles del procedimiento también se contemplan en contribuciones anteriores (12).

Para promover los procesos de interacción matriz/refuerzo, se han llevado a cabo tratamientos isotérmicos sobre los compuestos, a una temperatura de $530^{\circ} \mathrm{C}$ y a tiempos de permanencia de $1,6,12$ y $24 \mathrm{~h}$. Estos tratamientos se llevan a cabo en una atmósfera controlada y sobre muestras de $5 \mathrm{~mm}$ de espesor. 
La microestructura de los compuestos se ha estudiado sobre muestras preparadas metalográficamente por técnicas convencionales. Especial interés se ha prestado a la distribución del refuerzo en la matriz, la porosidad existente en los compuestos, así como el estudio de las interfaces matriz/refuerzo. El análisis microestructural se ha llevado a cabo mediante microscopía óptica MO (Nikon Microphot FX) y microscopía electrónica de barrido MEB (JEOL 6300). El microscopio electrónico, provisto de un dispositivo de microanálisis de rayos $\mathrm{X}$, ha permitido determinar de manera aproximada la estequiometría de la capa de reacción formada en las interfaces matriz/refuerzo. Además, se han utilizado técnicas de análisis de imagen para la determinación del espesor de la capa de reacción. Para ello se ha hecho uso del programa informático Visilog 5.0, el cual ha trabajado sobre imágenes tomadas en el MEB.

La presente investigación pretende evaluar la interacción matriz/ refuerzo al emplear partículas intermetálicas como refuerzo en una matriz de aluminio y al someter a los compuestos a un tratamiento térmico de solubilización. Se estudiará la capa de reacción formada en las interfaces matriz/refuerzo mediante análisis de imagen elaborando para ello una rutina adecuada y determinando el espesor de la capa formada. De esta forma se observará la influencia que el tipo y el contenido de refuerzo tienen en la interacción matriz/refuerzo.

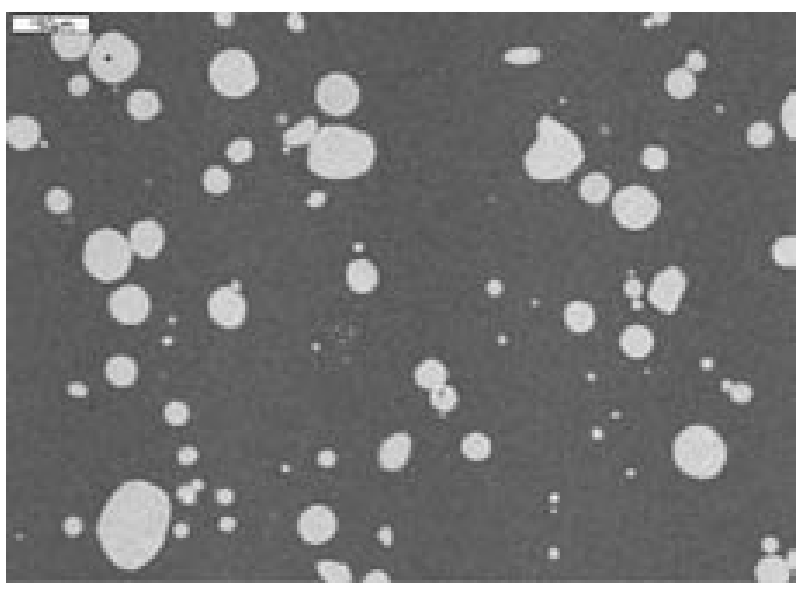

Figura 1. Sección transversal del compuesto con 10\% de TiAl.

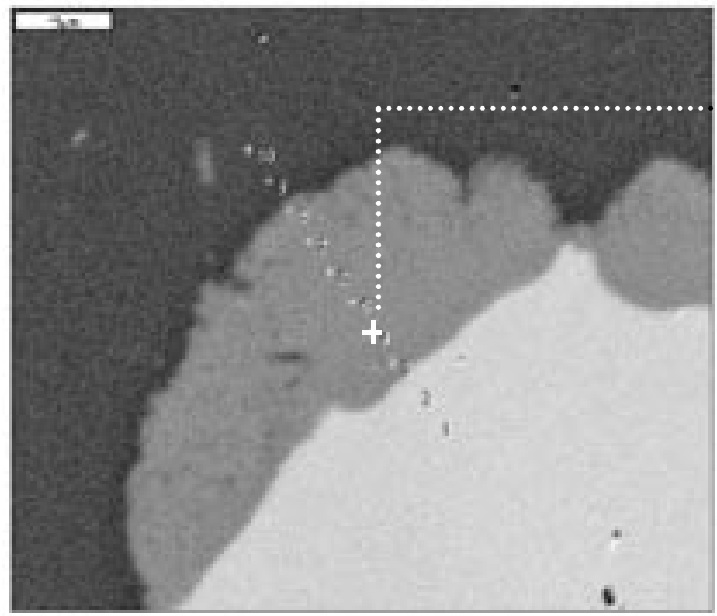

\section{RESULTADOS Y DISCUSIÓN}

El análisis microestructural de los compuestos reveló una distribución homogénea de las partículas intermetálicas en la matriz de aluminio en todos los compuestos fabricados, estando éstos prácticamente libres de porosidad, figura 1 .

Tras someter a las muestras al tratamiento térmico de solubilización, se observó transcurrido un tiempo, la formación de una capa de reacción en las interfaces matriz/refuerzo de los mismos.

Se realizaron diferentes estudios cualitativos y cuantitativos en las muestras para establecer la variación de los componentes presentes en la zona de reacción. Para este tipo de análisis se seleccionaron partículas con una sección lo más cercana a la teórica ecuatorial. El estudio cuantitativo de los elementos presentes en la capa de reacción, figura 2, permitió determinar las fases presentes en la misma. Los valores obtenidos se ajustaron a la estequiometría de las fases intermetálicas $(\mathrm{Al}, \mathrm{Si})_{2} \mathrm{Ti}$ y $(\mathrm{Al}, \mathrm{Si})_{3} \mathrm{Ti}$. Con el aumento del tiempo de solubilización, se observó la tendencia de la capa de reacción hacia la estabilización de la fase intermetálica ( $\mathrm{Al}, \mathrm{Si})_{3} \mathrm{Ti}$ que ocurrió a las 12 o 24 horas de solubilización, según el tipo de refuerzo intermetálico empleado. La observación de los espectros de análisis, verificó el importante incremento en la cantidad de silicio en esta nueva fase formada. Este silicio corresponde al silicio presente en la matriz no combinado con el magnesio formando el compuesto de equilibrio $\mathrm{Mg}_{2} \mathrm{Si}$ y que ha difundido hacia la capa de reacción.

Se intentó evaluar el crecimiento de la capa de reacción determinando para ello su espesor en cada uno de los compuestos ensayados. Con este fin se desarrollo una rutina de trabajo empleando un programa de análisis de imágenes. Dicha rutina se explica a partir del cálculo concreto en uno de los compuestos:

A. Determinación del número de partículas presentes en la muestra observada.

Para ello se toma una micrografía general de la muestra obtenida en el MEB a unos aumentos de X 250, figura 3. En el programa se ejecutan los siguientes comandos:

- Erosionar la imagen dos veces para observar la separación clara entre partículas, figura 4. Mediante esta herramienta informática, cada píxel perteneciente a una partícula es reemplazado por el valor de gris que sea mínimo de los píxeles inmediatamente vecinos. Como la escala de grises va desde el 0 (negro) al blanco (255), cada píxel en la vecindad de la matriz (de color negro) adquirirá el color de ésta, erosionándose efectivamente las partículas.

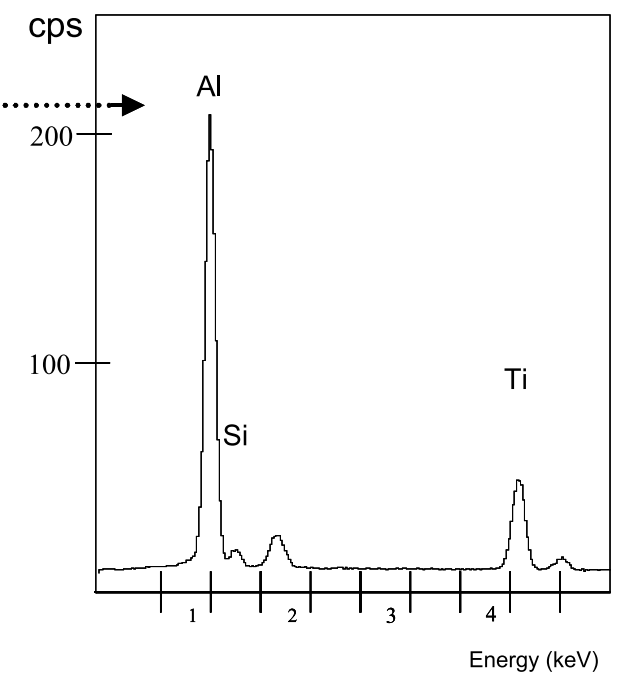

Figura 2. Análisis cuantitavo en la capa de reacción formada en el compuesto $5 \%$ de $\mathrm{Ti}_{3} \mathrm{Al}$ tras someterlo 24 horas a $530^{\circ} \mathrm{C}$. 


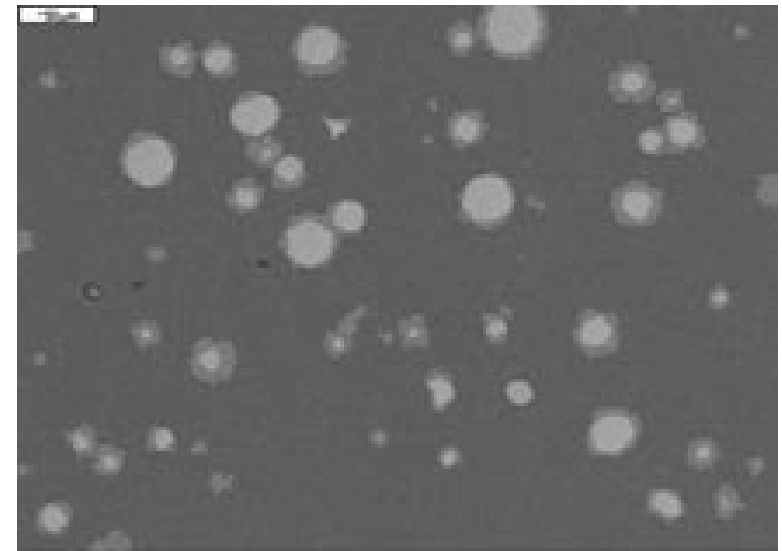

Figura 3. Micrografía del compuesto con $5 \%$ de $\mathrm{Ti}_{3} \mathrm{Al}$ tras $12 \mathrm{~h}$ a $530^{\circ} \mathrm{C}$.

- Dilatar la imagen dos veces. Mediante esta herramienta, que es la inversa de la anterior, se dilatan las partículas al reemplazar el valor de gris de cada píxel por el mayor (más claro) de sus inmediatos vecinos. De esta forma, la acción combinada de las operaciones de erosión y dilatación por este orden favorecen la eliminación de ruido en la imagen (pequeños puntos blancos no correspondientes con partículas) así como la separación de partículas adyacentes y de esta manera individualizarlas.

- Aplicar un análisis individual a una zona y en el visor ver el número total de partículas: en este caso concreto son 52 partículas.

B. Determinación del avance de reacción.

Para ello se procede del modo siguiente:

Se binariza la imagen con un intervalo de grises de 111-240, de manera que reducimos la imagen a dos colores correspondientes a la matriz (negro) y las partículas, lo que nos permitirá posteriormente calcular el área y perímetro total de grano (partícula + capa de reacción), figura 5 .

Se binariza la imagen con un intervalo de grises de 140-240, figura 6, lo que nos permitirá posteriormente calcular el área y perímetro central de grano (partícula).

De nuevo en el visor se pueden observar los valores calculados. Nos interesan el valor del perímetro total 7576 píxeles y el del perímetro central 5479 píxeles.

El valor del perímetro que está relacionado con el valor del radio nos lleva a obtener el valor del radio total $\mathbf{1 2 0 6}$ píxeles y el del radio central 872 píxeles.

Así el avance total de la capa de reacción viene dado por una simple sustracción, $1206-872=334$ píxeles. Haciendo uso de la regla de calibración utilizada que nos dice que 51 píxeles son $50 \mu \mathrm{m}$, se obtiene una avance total de reacción de $329 \mu \mathrm{m}$.

C. Determinación del avance medio por partícula (espesor de la capa).

La división del avance total de reacción por el número de partículas presentes en la muestra nos lleva a conocer el espesor medio de la capa de reacción por partícula individual: $6.3 \mu \mathrm{m}$.

Una vez calculados los espesores para cada una de las muestras en cada uno de los tratamientos isotérmicos realizados en ellas, se puede representar la tendencia observada para cada caso, figura 7, donde se representa el espesor frente al tiempo de solubilización. De esta gráfica se puede extraer que existen dos factores que condicionan la interacción matriz/refuerzo:

Contenido de refuerzo. A mayor contenido de refuerzo, el porcentaje de productos de reacción obtenido al aplicar técnicas de análisis de imagen a las micrografías obtenidas por SEM, es mayor en ambos refuerzos. Sin embargo considerando las partículas individualmente, el espesor de la capa de reacción es menor a mayor contenido de re-

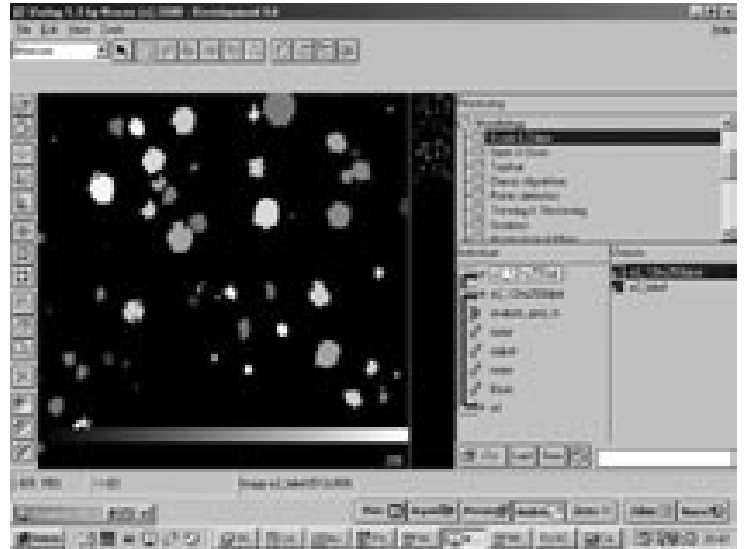

Figura 4. Ventana mostrada en el programa al erosionar dos veces la imagen y dilatarla otras dos.

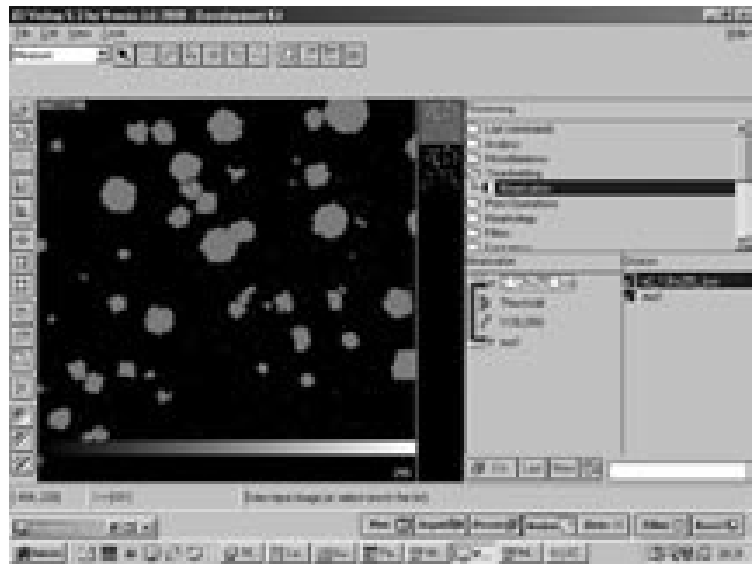

Figura 5. Ventana mostrada en el programa al binarizar con intervalo de grises de 111-240.

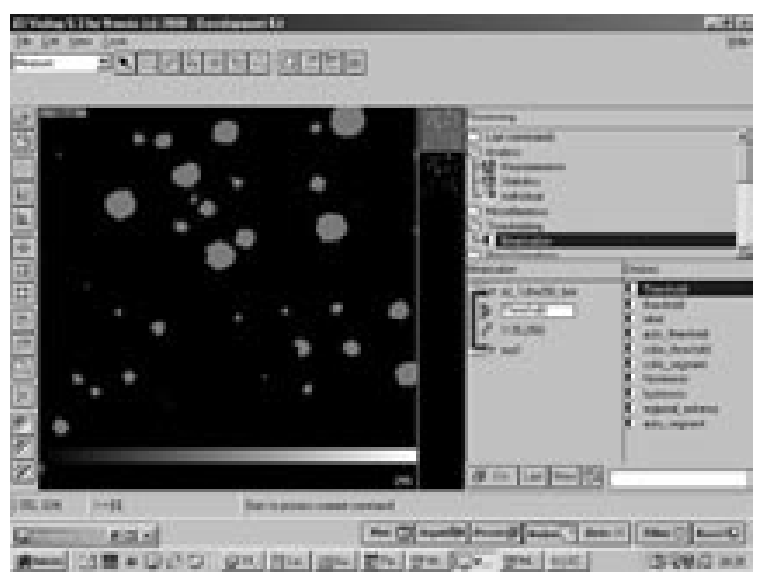

Figura 6. Ventana mostrada en el programa al binarizar con intervalo de grises de 141-240.

fuerzo, debido a que un mayor contenido de refuerzo en el material inhibe la difusión del Si presente en la matriz hacia la interface matriz/ refuerzo y por lo tanto aparece una menor cantidad de productos de reacción. Hecho que se comprueba también al observar las micrografías correspondientes a muestras con distintos contenidos de refuerzo y sometidas la mismo tratamiento térmico, figuras 8 y 9 .

Estabilidad del refuerzo. Se observa que para aquellas partículas intermetálicas que son más estables en el seno de la matriz de aluminio (partículas de TiAl), el espesor de capa de reacción formado a igual tratamiento térmico es menor, figuras 10 y 11 . Además, se puede comprobar que se mantiene esta estabilidad a altas temperaturas de tratamiento correspondientes a la solubilización. 


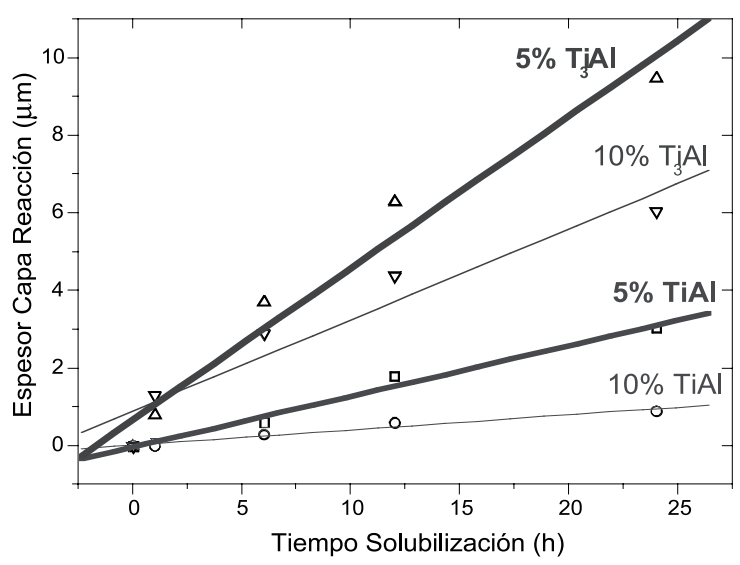

Figura 7. Espesor medido vs. tiempo de solubilización para cada uno de los compuestos

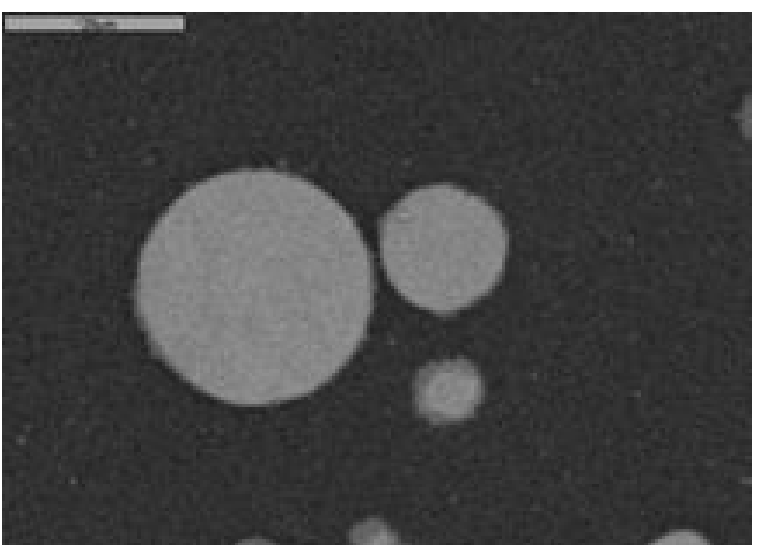

Figura 9. Compuesto con $10 \%$ de $\mathrm{TiAl}$ tratado $12 \mathrm{~h}$ a $530^{\circ} \mathrm{C}$.

\section{CONCLUSIONES}

A modo de resumen se puede decir:

La interacción matriz/refuerzo depende de la estabilidad del refuerzo intermetálico utilizado así como del contenido del mismo. Aumentando ambos, la interacción decrece.

El análisis de imágenes permite calcular con cierta precisión el espesor de las capas formadas.

\section{BIBLIOGRAFÍA}

1. T. S. Srivatsa \& I. A. Ibrahim F. A. Mohamed and E. J. Lavernia. Journal of Materials Science, Vol. 26, 5965-5978, (1991)

2. Numinium-Matrix Composites. Aluminium and Aluminium Alloys, ASM, 160-179, (1993).

3. D.J. Lloyd International Materials Reviews, Vol 39, 1-23 (1994)

4. B. C. Pai, G. -Ramani, R. M. Pillai and K.G. Satyanarayana. Journal of Materials Science, Vol 30, 1903-1911, (1995).

5. A. S. Chen, R. S. Bushby, M. G. Phillips \& V. D. Scott. Proc. R. Soc. London A, Vol 30, 1903-1911, (1995).

6. M. Yamada, Y. Unakoshi. Intermetallic Compounds, Nikkan Kogyo Press, Tokyo, Japan (1983).

7. Z. X. Guo, B. Derby. Prog. Material Science, Vol 39, 411, (1995).

8. F. L. Matthew, R. D. Rawlings. Composite Materials Engineering and Science. London, Champan \& Hall, (1994).

9. S. Bernath, T. Wagner, S. Hofmann, M. Rühle. Surface Science, 400, 553 (1998).

10. C. Ferrer, V. Amigó, M. D. Salvador, D. Busquets, J. M. Torralba. European Conference on Composite Materials ECCM-8, Vol. 4,67-74, (1998).

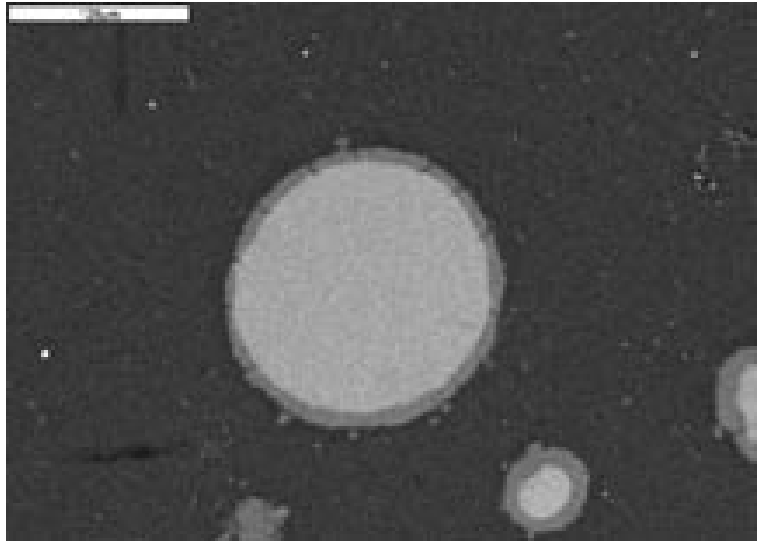

Figura 8. Compuesto con $5 \%$ de TiAl tratado $12 \mathrm{~h}$ a $530^{\circ} \mathrm{C}$

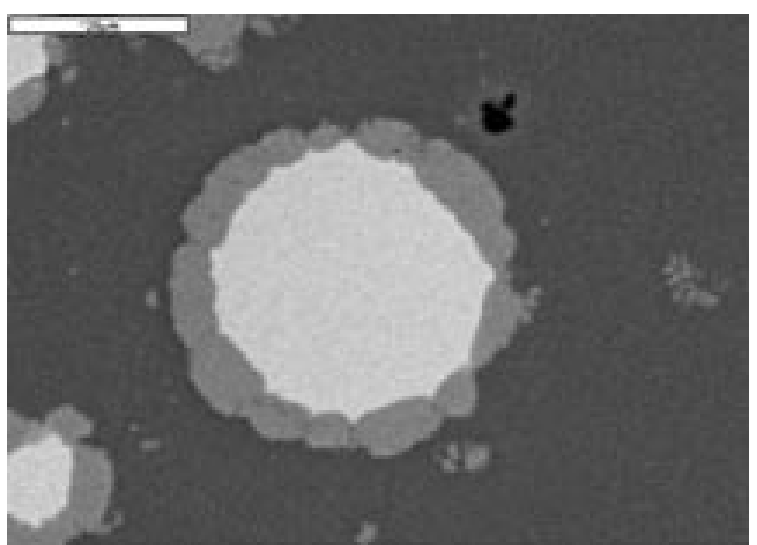

Figura 10. Compuesto con $5 \%$ de $\mathrm{Ti}_{3} \mathrm{Al}$ sometido $12 \mathrm{~h}$ a $530^{\circ} \mathrm{C}$

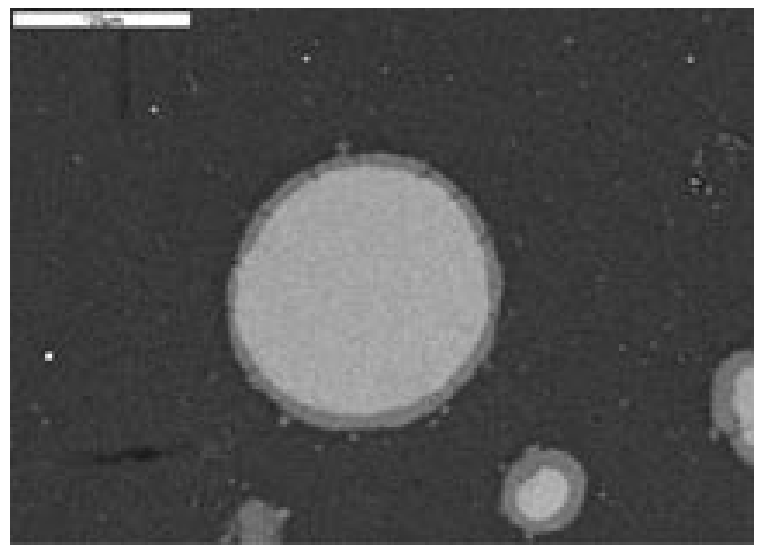

Figura 11. Compuesto con $5 \%$ de TiAl sometido $12 \mathrm{~h}$ a $530^{\circ} \mathrm{C}$

11. Spence-JCH. Materials Science and Engineering-Reports. Vol 26, 1, (1999). 12. M.D. Salvador, V. Amigó, N. Martinez, D.J. Busquets. AMPT '01 International Conference on Advances in Materials and Processing Technologies, Madrid (2001).

Recibido: 1.2 .03

Aceptado: 30.11 .03 